\title{
UNTWISTING INFORMATION FROM HEEGAARD FLOER HOMOLOGY
}

\author{
KENAN INCE
}

\begin{abstract}
The unknotting number of a knot is the minimum number of crossings one must change to turn that knot into the unknot. We work with a generalization of unknotting number due to Mathieu-Domergue, which we call the untwisting number. The $p$-untwisting number is the minimum number (over all diagrams of a knot) of full twists on at most $2 p$ strands of a knot, with half of the strands oriented in each direction, necessary to transform that knot into the unknot. In previous work, we showed that the unknotting and untwisting numbers can be arbitrarily different. In this paper, we show that a common route for obstructing low unknotting number, the Montesinos trick, does not generalize to the untwisting number. However, we use a different approach to get conditions on the Heegaard Floer correction terms of the branched double cover of a knot with untwisting number one. This allows us to obstruct several 10 and 11-crossing knots from being unknotted by a single positive or negative twist. We also use the Ozsváth-Szabó tau invariant and the Rasmussen $s$ invariant to differentiate between the $p$ - and $q$-untwisting numbers for certain $p, q>1$.
\end{abstract}

\section{INTRODUCTION}

It is a natural knot-theoretic question to seek to measure "how knotted up" a knot is. One such "knottiness" measure is given by the unknotting number $u(K)$, the minimum number of crossings, taken over all diagrams of $K$, one must change to turn $K$ into the unknot. By a crossing change we shall mean one of the two local moves on a knot diagram given in Figure 1.1.

This invariant is quite simple to define but has proven itself very difficult to master. Fifty years ago, Milnor conjectured that the unknotting number for the $(p, q)$-torus knot was $(p-1)(q-1) / 2$; only in 1993, in two celebrated papers [KM93, KM95], did Kronheimer and Mrowka prove this conjecture true. Hence, it is desirable to look at variants of unknotting number which may be more tractable. One natural variant (due to Murakami [Mur90]) is the algebraic unknotting number $u_{a}(K)$, the minimum number of crossing changes necessary to

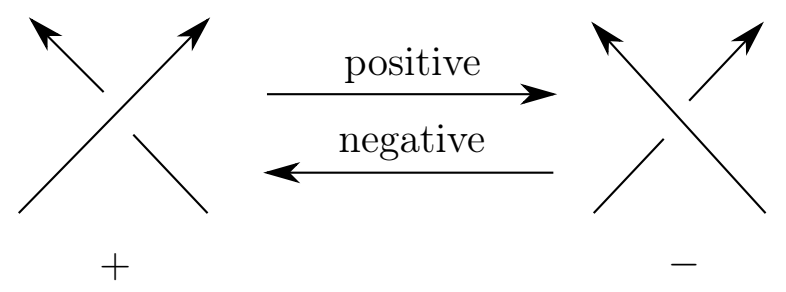

Figure 1.1. A positive and negative crossing change. 
turn a given knot into an Alexander polynomial-one knot. Alexander polynomial-one knots are significant because they "look like the unknot" to classical invariants, knot invariants derived from the Seifert matrix. It is obvious that $u_{a}(K) \leq u(K)$ for any knot $K$, and there exist knots such that $u_{a}(K)<u(K)$ (for instance, the Whitehead double of any nontrivial knot).

In [MD88], Mathieu and Domergue defined another generalization of unknotting number. In [Liv02], Livingston worked with this definition. He described it as follows:

"One can think of performing a crossing change as grabbing two parallel strands of a knot with opposite orientation and giving them one full twist. More generally, one can grab $2 k$ parallel strands of $K$ with $k$ of the strands oriented in each direction and give them one full twist."

Following Livingston, we call such a twist a generalized crossing change. We describe in [Inc16] how a crossing change may be encoded as a \pm 1 -surgery on a nullhomologous unknot $U \subset S^{3}-K$ bounding a disk $D$ such that $D \cap K=2$ points. From this perspective, a generalized crossing change is a relaxing of the previous definition to allow $D \cap K=2 k$ points for any $k$, provided $\operatorname{lk}(K, U)=0$ (see Fig. 1.2). In particular, any knot can be unknotted by a finite sequence of generalized crossing changes.
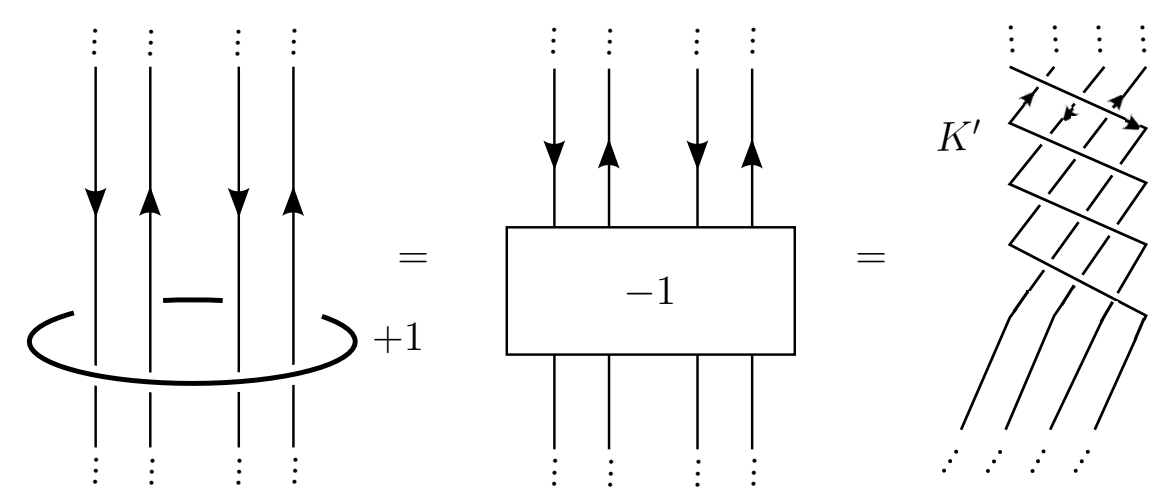

Figure 1.2. A left-handed, or positive, generalized crossing change.

One may then naturally define the untwisting number $t u(K)$ to be the minimum length, taken over all diagrams of $K$, of a sequence of generalized crossing changes beginning at $K$ and resulting in the unknot. By $t u_{p}(K)$, we will denote the minimum number of generalized crossing changes on $2 p$ or fewer strands, with $p$ strands oriented in each direction, needed to unknot $K$. Notice that $t u_{1}=u$ and that

$$
t u \leq \cdots \leq t u_{p+1} \leq t u_{p} \leq \cdots \leq t u_{1}=u
$$

The algebraic untwisting number $t u_{a}(K)$ is the minimum number of generalized crossing changes, taken over all diagrams of $K$, needed to transform $K$ into an Alexander polynomialone knot. It is clear that $t u_{a}(K) \leq t u(K)$ for all knots $K$. In [Inc16], we showed that, in fact, $t u_{a}(K)=u_{a}(K)$ for all knots $K$, hence the unknotting and untwisting numbers are "algebraically the same". However, we also showed that $t u$ and $u$ can be arbitrarily different in general: there exists a family of knots $\left\{S_{p}^{q}\right\}$ such that $\left(u-t u_{q}\right)\left(S_{p}^{q}\right) \geq p-1$ for all $p, q \geq 2$. 
Since the family $\left\{S_{p}^{q}\right\}$ consists of $(p, 1)$-cables of (untwisted) Whitehead doubles, most members of this family have very high crossing number. In this paper, we compare the unknotting and untwisting numbers for several 10 and 11-crossing knots with signature 0 . In order to do this, we will develop an obstruction to a knot with signature 0 having untwisting number 1. This will require the methods of Heegaard Floer homology, specifically the $d$-invariants or Heegaard Floer correction terms of a 3-manifold.

In [OS05], Ozsváth and Szabó develop an unknotting number 1 obstruction using $d$-invariants. This obstruction relies on the Montesinos trick, which allows them to construct a definite 4-manifold with boundary the branched double cover $\Sigma(K)$ of an unknotting number-1 knot $K$. In Section 3, we give an infinite family of knots which have untwisting number 1 but which do not satisfy the Montesinos trick, eliminating that route toward a $d$-invariant obstruction:

Theorem 1.1. There exists an infinite family $\left\{K_{n}\right\}_{n>1}$ of knots such that $t u\left(K_{n}\right)=1$ for all $n$, but $\Sigma\left(K_{n}\right)$ is not half-integer surgery on any knot in $S^{3}$ for any $n$.

In Section 4, we get around the failure of the Montesinos trick for untwisting number-1 knots by porting the machinery used by Owens and Strle in [OweS13] and Nagel and Owens in [NO15] as an obstruction to low untwisting number:

Theorem 1.2. Let $K$ be a knot with signature $\sigma(K)$ which can be unknotted by positive and $n$ negative generalized crossing changes. Then $Y=\Sigma(K)$, the branched double cover of $K$, bounds a smooth, definite 4-manifold $W$ with $b_{2}(W)=2 n+2 p$ and signature $2 n-2 p+$ $\sigma(K)$. Moreover, $H_{2}(W ; \mathbb{Z})$ contains $n$ classes of self-intersection +2 and $p$ classes of selfintersection -2 which span a primitive sublattice; in other words, the quotient of $H_{2}(W ; \mathbb{Z})$ by this sublattice is torsion-free.

Once we have constructed a definite 4-manifold $W$ with $\partial W=\Sigma(K)$, the next step is to apply a result of Ozsváth-Szabó to get conditions that the $d$-invariants of $\Sigma(K)$ must satisfy. These invariants are easily computable for alternating $K$ via the Goeritz matrix associated to $K$. These computations are discussed further in Section 4. We successfully obstruct several 10-crossing knots from being unknotted by a single positive and/or negative generalized crossing change, though these untwisting numbers cannot be computed using the methods available prior to the development of Heegaard Floer homology:

Theorem 1.3. The knots $10_{68}$ and $10_{96}$ have untwisting number 2 ; the knots $10_{22}, 10_{34}$, $10_{35}, 10_{87}$, and $10_{90}$ cannot be unknotted by a single positive generalized crossing change; and the knot $10_{48}$ cannot be unknotted by a single negative generalized crossing change.

Similarly, we apply these obstructions to all 11-crossing knots with signature 0, algebraic unknotting number 1 , and unknotting number 2 to get the following:

Theorem 1.4. The knots $11 a_{37}, 11 a_{103}, 11 a_{169}, 11 a_{214}$, and $11 a_{278}$ have untwisting number 2 .

Finally, we showed in [Inc16] that there can be arbitrarily large gaps between the $p$-untwisting number and the 1-untwisting number (which by definition equals the unknotting number) for several families of knots. However, we had not yet been able to distinguish between $t u_{p}$ and $t u_{q}$ for $p, q>1$. 
In Section 6, we use invariants coming from Heegaard Floer homology (the Ozsváth-Szabó $\tau$ invariant) and Khovanov homology (the Rasmussen $s$ invariant) to give lower bounds on the $p$-untwisting number for arbitrary $p$ via the following theorem. While visiting Mark Powell at the Max Planck Institute, he suggested this theorem and told me an outline of a proof similar to the proof of Powell and coauthors T. Cochran, S. Harvey, and A. Ray that the $\tau$ and $s$ invariants give lower bounds for their bipolar metrics (to appear in a future paper). An anonymous referee suggested a simpler approach involving the 4-genus, detailed in section 6.

Theorem 1.5. Let $K$ be a knot which can be converted to the unknot via $n$ generalized crossing changes, where for every $i$, the ith generalized crossing change is performed on $2 p_{i}$ strands. Then

$$
|\tau(K)| \leq \sum_{i=1}^{n} p_{i}^{2}
$$

and

$$
\frac{|s(K)|}{2} \leq \sum_{i=1}^{n} p_{i}^{2}
$$

This allows us to show that there exist $p, q>1$ so that the difference between the $p$ - and $q$-untwisting numbers of several families of knots can be made arbitrarily large:

Example 1.6. Let $K_{p^{3}}$ denote the $\left(p^{3}, 1\right)$-cable of a knot $K$ with genus 1 and $u(K)=1=$ $\tau(K)$ (one example of such a $K$ is the right-handed trefoil knot). We know from [Inc16, Section 5] that $t u_{p^{3}}\left(K_{p^{3}}\right)=1$. We may use Theorem 1.5 to show that

$$
t u_{p}\left(K_{p^{3}}\right)-t u_{p^{3}}\left(K_{p^{3}}\right) \stackrel{p \rightarrow \infty}{\longrightarrow} \infty .
$$

Convention. In this paper, all manifolds are assumed to be smooth, compact, orientable, and connected, and all surfaces in manifolds are assumed to be smoothly embedded. When homology groups are given without specifying coefficients, they are assumed to have coefficients in $\mathbb{Z}$.

Acknowledgement. Thanks to Stefan Friedl, Maciej Borodzik, Peter Horn, Matthias Nagel, and Mark Powell for many enlightening conversations. Thanks also to Stefan Friedl, Matthias Nagel, Brendan Owens, and an anonymous referee for providing comments on this paper. A Maple program written by Brendan Owens and Sašo Strle has been very useful in the computations in Section 5. I would also like to acknowledge the results of Brendan Owens and Sašo Strle in [OweS13], Matthias Nagel and Brendan Owens in [NO15], Brendan Owens in [Owe08], and Tim Cochran and William Lickorish in [CL86], all of which greatly inspired this work.

\section{Preliminaries}

2.1. Dehn surgery. In this section, we will describe the operation of Dehn surgery on knots. 
Definition 2.1. Let $K \subset S^{3}$ be an oriented knot, let $N$ be a closed tubular neighborhood of $K$, and consider the preferred framing for $N$ (see [Rol76, Definition 2E8]) in which the longitude $L$ is oriented in the same way as $K$ and the meridian $M$ has linking number +1 with $K$. We may write any simple closed curve $J \subset \partial N$ in terms of the homology basis $\{\lambda=[L], \mu=[M]\}$ :

$$
[J]=q \lambda+p \mu \in H_{1}(\partial N)
$$

The result of $\frac{p}{q}$-surgery on $K$ is the 3 -manifold

$$
S_{p / q}^{3}(K):=\left(S^{3}-\stackrel{\circ}{N}\right) \bigcup_{h}\left(S^{1} \times D^{2}\right),
$$

where $h: \partial\left(S^{1} \times D^{2}\right) \rightarrow \partial N$ is a homeomorphism taking $* \times S^{1}$ onto a curve $J$ of class $[J]=p \mu+q \lambda$ in $H_{1}(\partial N)$. By convention, we indicate that surgery is to be performed on $K$ by writing the ratio $\frac{p}{q}$ next to a diagram of $K$.

If $U \subset S^{3} \backslash K$ is an unknot such that $\operatorname{lk}(K, U)=0$, we define a generalized crossing change diagram for $K$ to be a diagram of the link $K \sqcup U$ with the number \pm 1 written next to $U$, indicating that $U$ is to have \pm 1 -surgery performed on it.

There is an orientation-preserving homeomorphism $\Phi$ of the manifold $M:=S_{ \pm 1}^{3}(U)$ resulting from \pm 1 -surgery on $U$ with $S^{3}$. However, $K^{\prime}:=\Phi(K) \subset S^{3}$ may have a different knot type than $K$. (Note that the knot type of $K^{\prime}$ does not depend on the choice of homeomorphism $\Phi$ since any two orientation-preserving homeomorphisms of $S^{3}$ are isotopic.) In particular, if $D$ is a disk bounded by $U$ such that $2 p$ strands of $K$ pass through $D$ in straight segments, then each of the $2 p$ straight pieces is replaced by a helix which screws through a neighborhood of $D$ in the right- (respectively, left-) hand sense (see Fig. 2.1).

The process of performing \pm 1 -surgery on an unknot $U$ in a generalized crossing change diagram for a knot $K$, mapping the resulting manifold to $S^{3}$ via an orientation-preserving homeomorphism $\Phi$, then erasing $\Phi(U)$ from the resulting diagram of $\Phi(K) \sqcup \Phi(U)$ is called a \pm -generalized crossing change on $K$. Now, it can be easily verified that performing a --generalized crossing change on the knot $K$ on the left side of Figure 2.2 transforms the crossing labeled + into the crossing labeled - . The inverse process of introducing an unknot labeled with $\mathrm{a}+1$ to the right side of Figure 2.2 and performing a + -generalized crossing change in the resulting generalized crossing change diagram transforms the crossing labeled - into the crossing labeled + .

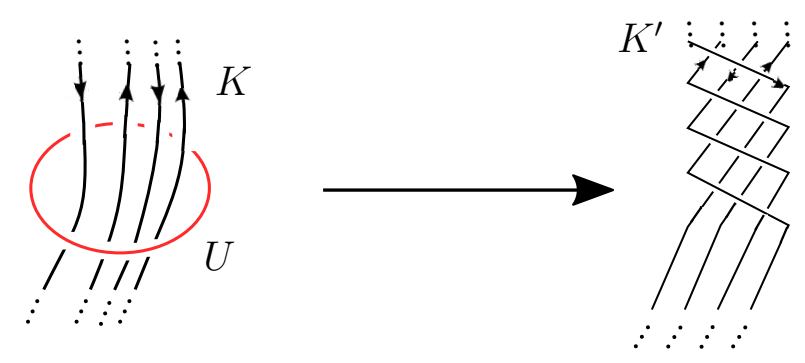

Figure 2.1. Performing +1 -surgery on an unknot $U$ gives the knot $K$ a lefthanded twist. 


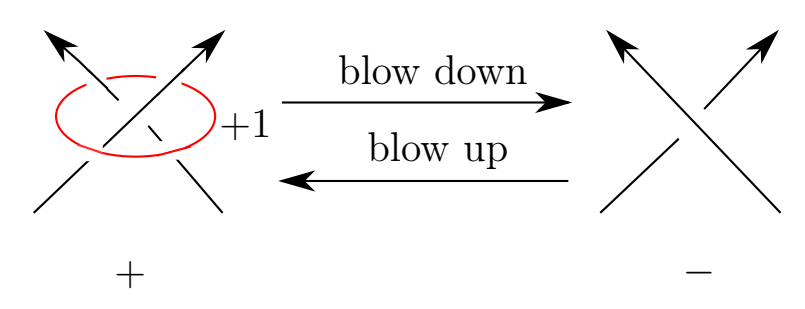

Figure 2.2. A crossing change is a 1-generalized crossing change.

2.2. The untwisting number. In a generalized crossing change diagram for $K$ consisting of a diagram of $K$ and an unknot $U, K$ must pass through $U$ an even number of times, for otherwise $\operatorname{lk}(K, U) \neq 0$. If at most $2 p$ strands of $K$ pass through an unknot $U$ in a generalized crossing change diagram, we may call the associated \pm -generalized crossing change a $\pm p$-generalized crossing change on $K$.

The untwisting number tu(K) of $K$ is the minimum length of a sequence of generalized crossing changes on $K$ such that the result of the sequence is the unknot, where we allow ambient isotopy of the diagram in between generalized crossing changes. Note that by the reasoning on page 58 of [Ada94], this definition is equivalent to taking the minimum length, over all diagrams of $K$, of a sequence of generalized crossing changes beginning with a fixed diagram of $K$ such that the result of the sequence is the unknot, where we do not allow ambient isotopy of the diagram in between generalized crossing changes.

For $p=1,2,3, \ldots$, we define the $p$-untwisting number $t u_{p}(K)$ to be the minimum length of a sequence of $\pm p$-generalized crossing changes on $K$ resulting in the unknot, where we allow ambient isotopy of the diagram in between generalized crossing changes. It follows immediately that we have the chain of inequalities

$$
t u(K) \leq \cdots \leq t u_{p+1}(K) \leq t u_{p}(K) \leq \cdots \leq t u_{2}(K) \leq t u_{1}(K)=u(K) .
$$

2.3. Heegaard Floer homology. In this section, we will recall some properties of Heegaard Floer homology, a set of invariants of 3-manifolds defined by Ozsváth and Szabó. For details, refer to their papers, in particular [OS03a, OS03b, OS05].

Let $Y$ be an oriented rational homology 3-sphere. Recall that one can associate to $Y$ a set $\operatorname{Spin}^{c}(Y)$ of $\operatorname{spin}^{c}$ structures on $Y$. In the case where $\left|H^{2}(Y ; \mathbb{Z})\right|$ is odd, there is a canonical bijection $H^{2}(Y ; \mathbb{Z}) \leftrightarrow \operatorname{Spin}^{c}(Y)$ under which $0 \in H^{2}(Y ; \mathbb{Z})$ is sent to the unique spin structure on $Y$. In this way, we may give $\operatorname{Spin}^{c}(Y)$ a group structure inherited from that of $H^{2}(Y ; \mathbb{Z})$.

Fix a $\operatorname{spin}^{c}$ structure $\mathfrak{s}$ on $Y$. Then the (plus flavor of) Heegaard Floer homology $\mathrm{HF}^{+}(Y, \mathfrak{s})$ is a $\mathbb{Q}$-graded abelian group with a $\mathbb{Z}[U]$-action, where multiplication by $U$ lowers the grading by 2. Associated to $\mathfrak{s}$ is a $d$-invariant $d(Y, \mathfrak{s}) \in \mathbb{Q}$ which satisfies the symmetry condition $d(Y, \mathfrak{s})=-d(-Y, \mathfrak{s})$. The correction terms are useful for obstructing the existence of a 4-manifold with boundary $Y$ :

Theorem 2.2 (Ozsváth-Szabó 2003, [OS03a]). Let X be a negative definite 4-manifold with boundary $Y$ and intersection form represented by a matrix $Q$, and let $\mathfrak{s}$ be any spin ${ }^{c}$ structure $^{-}$ 
on $X$. Let $c_{1}(\mathfrak{s})$ denote the first Chern class associated to $\mathfrak{s}$. Then

$$
\begin{aligned}
\frac{c_{1}(\mathfrak{s})^{2}+b_{2}(X)}{4} & \leq d\left(Y,\left.\mathfrak{s}\right|_{Y}\right) \\
\text { and } \frac{c_{1}(\mathfrak{s})^{2}+b_{2}(X)}{4} & \equiv d\left(Y,\left.\mathfrak{s}\right|_{Y}\right) \quad \bmod 2 .
\end{aligned}
$$

Following [OS03a], we now show how to check this obstruction in practice. In addition to the assumptions of Theorem 2.2, suppose for simplicity that $\pi_{1}(X)=1$ and that $\left|H^{2}(Y ; \mathbb{Z})\right|$ is odd. (This will always be true for the examples in this paper.) Let $r=b_{2}(X)$, the second Betti number of $X$. It is straightforward to see that $H_{2}(X ; \mathbb{Z})$ is free of rank $r$ in this case. Choose a basis $\left\{x_{i}\right\}_{i=1}^{r}$ for $H_{2}(X ; \mathbb{Z})$ and let $Q=\left(Q_{i j}\right)$ be a negative definite $r \times r$ matrix representing the intersection pairing of $X$ in this basis; then $\operatorname{det} Q=\left|H^{2}(Y ; \mathbb{Z})\right|$. The dual basis $\left\{x^{i}\right\}_{i=1}^{r}$ for $H^{2}(X ; \mathbb{Z})$ given by the Universal Coefficient Theorem defines an isomorphism $H^{2}(X ; \mathbb{Z}) \cong \mathbb{Z}^{r}$. Under this isomorphism, the set $\left\{c_{1}(\mathfrak{s}) \mid \mathfrak{s} \in \operatorname{Spin}^{c}(X)\right\} \subset$ $H^{2}(X ; \mathbb{Z})$ of first Chern classes of $\operatorname{spin}^{c}$ structures on $X$ is sent to the set of characteristic covectors $\operatorname{Char}(Q)$ for $Q$. (Recall that a characteristic covector for an $r \times r$ matrix $Q$ is a vector $\xi=\left(\xi_{1}, \ldots, \xi_{r}\right) \in \mathbb{Z}^{r}$ such that $\xi_{i} \equiv Q_{i i} \bmod 2$ for $i=1, \ldots, r$.) In our basis, the square of the first Chern class of the $\operatorname{spin}^{c}$ structure corresponding to a characteristic covector $\xi$ is given by $\xi^{T} Q^{-1} \xi$.

Define a function $m_{Q}: \mathbb{Z}^{r} / Q\left(\mathbb{Z}^{r}\right) \rightarrow \mathbb{Q}$ by

$$
m_{Q}(g)=\max \left\{\frac{\xi^{T} Q^{-1} \xi+r}{4} \mid \xi \in \operatorname{Char}(Q),[\xi]=g\right\}
$$

where $[\xi]$ is the image of $\xi \in \mathbb{Z}^{r}$ under the projection to $\mathbb{Z}^{r} / Q\left(\mathbb{Z}^{r}\right)$. In computing $m_{Q}$, it is enough to consider characteristic covectors $\xi=\left(\xi_{1}, \ldots, \xi_{r}\right)$ with $-Q_{i i} \geq \xi_{i} \geq Q_{i i}$; if, say, $\xi_{i}<Q_{i i}$, subtracting twice the $i$ th column of $Q$ from $\xi$ shows that $\xi^{T} Q^{-1} \xi$ is not maximal. Then we may simplify the conditions (2.2) as follows:

Theorem 2.3 (Ozsváth-Szabó). Let $Y$ be a rational homology 3-sphere which is the boundary of a simply connected, negative definite 4-manifold $X$, with $\left|H^{2}(Y ; \mathbb{Z})\right|$ odd. If the intersection pairing of $X$ is represented in a basis by the matrix $Q$, then there is a group isomorphism

$$
\phi: \mathbb{Z}^{r} / Q\left(\mathbb{Z}^{r}\right) \rightarrow \operatorname{Spin}^{c}(Y)
$$

such that

$$
\begin{aligned}
& m_{Q}(g) \leq d(Y, \phi(g)) \\
& m_{Q}(g) \equiv d(Y, \phi(g)) \quad \bmod 2
\end{aligned}
$$

for all $g \in \mathbb{Z}^{r} / Q\left(\mathbb{Z}^{r}\right)$.

Under the assumptions of the theorem, we say that the 4-manifold $X$ bounded by $Y$ is sharp if equality holds in (2.3). In this case, we may compute the correction terms for $Y$ using the values of $m_{Q}$. Moreover, if a rational homology sphere $Y$ bounds a positive definite 4-manifold $X$, we may compute the correction terms for $Y$ by applying Theorem 2.3 to $-Y$.

If $K$ is an alternating knot, we may compute the $d$-invariants of $\Sigma(K)$ using the negative definite Goeritz matrix computed from an alternating diagram of $K$ as follows. Consider 
a regular projection of $K$ into a plane $\mathbb{R}^{2} \subset \mathbb{R}^{3}=S^{3} \backslash\{\infty\}$. Color the regions of $\mathbb{R}^{2} \backslash K$ alternately black and white so that the $n$ white regions $X_{1}, \ldots, X_{n}$ are separated by crossings of the type depicted in the figure below.

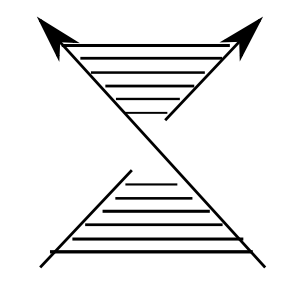

FiguRE 2.3. Crossing conventions for negative definite Goeritz matrices of alternating knots.

For $0 \leq i, j \leq n$, define

$$
g_{i j}=\left\{\begin{array}{ll}
d, \text { where } d \text { is the number of double points incident to } X_{i} \text { and } X_{j}, & i \neq j \\
-\sum_{k \neq i} g_{i k}, & i=j .
\end{array} .\right.
$$

Let $G^{\prime}=\left(g_{i j}\right)$. Then the negative definite Goeritz matrix $G$ associated to $K$ is the $n \times n$ symmetric integer matrix obtained from $G^{\prime}$ by deleting the 0 th row and column of $G^{\prime}$. It is shown in [OS05, Proposition 3.2] that $G$ represents the intersection pairing of a sharp 4manifold with boundary $\Sigma(K)$; thus, the correction terms for $\Sigma(K)$ are given by the values of $m_{G}$.

\section{Failure of the Montesinos trick}

The "Montesinos trick" relates crossing changes downstairs on $K$ to surgery upstairs on $\Sigma(K)$, the branched double cover of $K$. We use the convention that the determinant of a knot is given by $\left|\Delta_{K}(-1)\right|$, where $\Delta_{K}$ is the Alexander polynomial for the knot $K$.

Theorem 3.1. [Mon76] If $u(K)=1$, then $\Sigma(K) \cong S_{ \pm D / 2}^{3}(C)$ for some other knot $C \subset S^{3}$, where here $D$ is the determinant of $K$.

We show that this theorem does not generalize to untwisting number-1 knots:

Theorem 3.2. There exists an infinite family $\left\{K_{n}\right\}$ of knots such that, for all $n \geq 1$, tu $\left(K_{n}\right)=1$, but $\Sigma\left(K_{n}\right)$ is not half-integer surgery on any knot in $S^{3}$.

In order to prove Theorem 3.2, we will need two main ingredients. The first is the following lemma:

Lemma 3.3. The effect of performing a +-generalized crossing change on the unknot $U$ in the local picture given in Figure 3.1 is to add -4 full twists to the knot $K$.

Proof. See Figure 3.1. The intermediate steps are left to the reader.

Our second ingredient is the following theorem of McCoy [McC13]:

Theorem 3.4. Let $K$ be an alternating knot. Then the following are equivalent: 

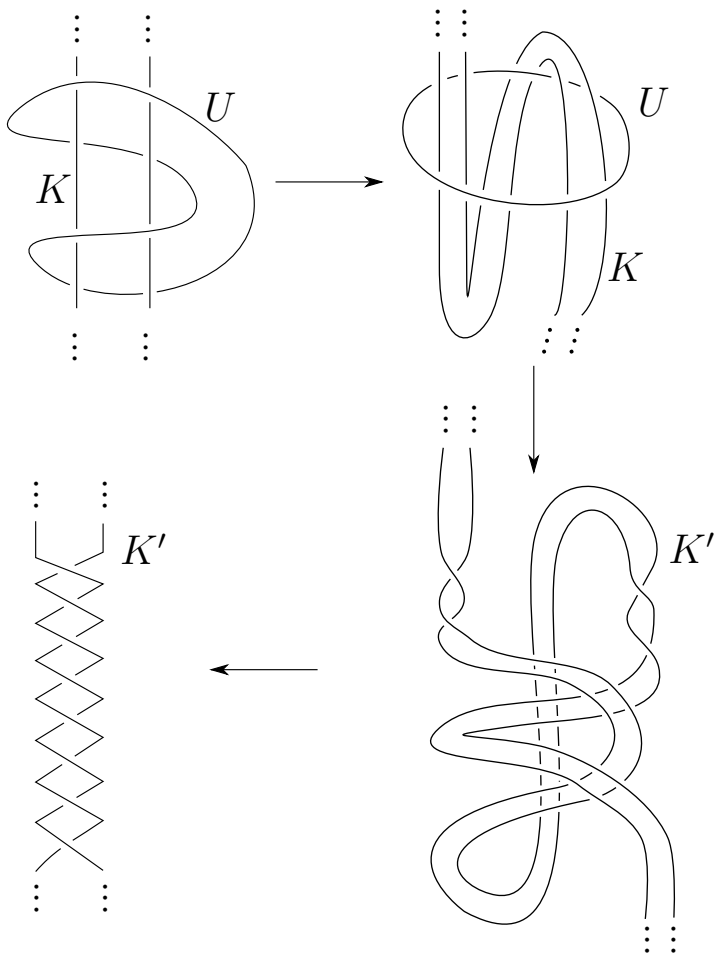

FiguRE 3.1. The (local) effect of performing a +-generalized crossing change on the unknot $U$.

(1) $u(K)=1$;

(2) the branched double cover $\Sigma(K)$ can be obtained by half-integer surgery on some knot in $S^{3}$;

(3) in every minimal diagram of $K$, there exists a crossing which can be changed to unknot that diagram.

Proof of Theorem 3.2. Fix an orientation on $K_{n}$. The generalized crossing change pictured in Figure 3.2 introduces -4 twists on the left side of $K_{n}$, which undo the 4 twists already present. Hence, $t u\left(K_{n}\right)=1$ for all $n$. Moreover, if $n>1$, then $K_{n}$ is a minimal diagram of an alternating knot. One can easily see that $K_{n}$ cannot be unknotted by any single crossing change in this diagram. By Theorem 3.4, the branched double cover $\Sigma\left(K_{n}\right)$ cannot be obtained by half-integer surgery on any knot in $S^{3}$, and moreover $u\left(K_{n}\right)>1$.

Note 3.5. The first knot in this family is $K_{2}=12 a_{1166}$. The unknotting number of $12 a_{1166}$ is listed as "not known" in the KnotInfo tables, but is either 1 or 2. By Theorem 3.4, we must have that $t u\left(12 a_{1166}\right)=1<2=u\left(12 a_{1166}\right)$.

Question 3.6. Does there exist a knot $K$ with $t u(K)=1$ such that $\Sigma(K)$ is not surgery on any knot in $S^{3}$ ? 


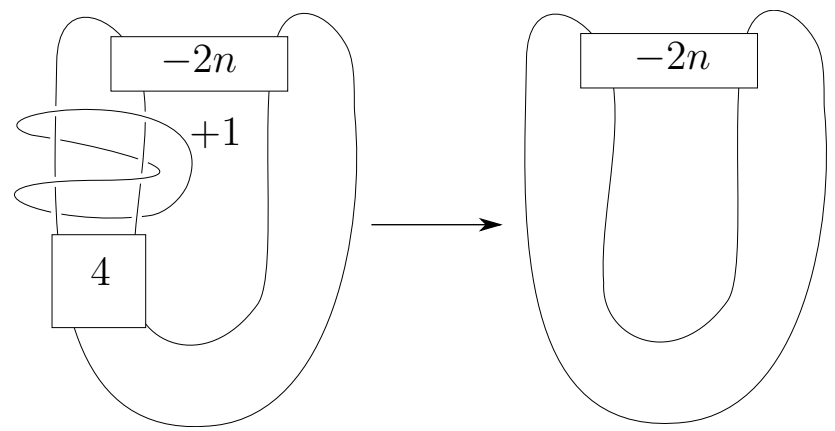

Figure 3.2. The knots $K_{n}$, together with the +1 -generalized crossing change that unknots them. Here, positive (resp., negative) numbers in boxes denote right-handed (resp., left-handed) full twists.

\section{Heegaard Floer-theoretic obstructions to untwisting number 1}

Although the Montesinos trick does not hold for knots with untwisting number 1, we can still get obstructions to a knot $K$ being unknotted by a single positive or negative generalized crossing change using techniques similar to those of Owens and Strle in [OweS13] and Nagel and Owens in [NO15] together with Theorem 2.2.

In order to apply Theorem 2.2, we first compute a Goeritz matrix $G$ for $K$ and, from $G$, the function $m_{G}$ as in Proposition 2.2. The image of $\mathbb{Z}^{r} / G\left(\mathbb{Z}^{r}\right)$ under $m_{G}$, where $G$ is an $r \times r$ matrix, is the set of $d$-invariants for $Y$. We construct the 4-manifold $W$ as in [NO15, Proposition 2.3] using the propositions below, then compute the $m_{Q}$ and show that no isomorphism satisfying both of the conditions (2.2) exists.

Proposition 4.1. Let $K$ be an oriented knot in $S^{3}$, and suppose that $K$ can be unknotted by $p$ positive and $n$ negative generalized crossing changes. Then $K$ bounds a disk $\Delta$ in $a$ manifold $C \cong B^{4} \#_{n} \mathbb{C P}^{2} \#_{p} \overline{\mathbb{C P}^{2}}$ with $[\Delta]=0 \in H_{2}(C, \partial C)$ and $\pi_{1}(C \backslash \Delta)=\mathbb{Z}$, generated by a meridian of $K$.

Proof. Suppose that $K$ is an oriented knot in $S^{3}$ and that $K$ can be unknotted by $p$ positive and $n$ negative generalized crossing changes. Then there is a sequence of knots

$$
K:=K_{p+n} \stackrel{\epsilon_{p+n}}{\longrightarrow} K_{p+n-1} \stackrel{\epsilon_{p+n-1}}{\longrightarrow} \ldots \stackrel{\epsilon_{2}}{\longrightarrow} K_{1} \stackrel{\epsilon_{1}}{\rightarrow} K_{0}:=U
$$

for which $K_{i}$ is obtained from $K_{i+1}$ by a single generalized crossing change of sign $\epsilon_{i+1} \in\{ \pm 1\}$ for $i=1, \ldots, p+n$, with precisely $p$ of the $\epsilon_{i}$ equal to +1 and $n$ of the $\epsilon_{i}$ equal to -1 , and $U$ is the unknot. Reversing our point of view, there is a sequence of knots

$$
U:=K_{0} \stackrel{-\epsilon_{1}}{\longrightarrow} K_{1} \stackrel{-\epsilon_{2}}{\longrightarrow} \ldots \stackrel{-\epsilon_{p+n-1}}{\longrightarrow} K_{p+n-1} \stackrel{-\epsilon_{p+n}}{\longrightarrow} K_{p+n}=: K
$$

for which $K_{i}$ is obtained from $K_{i-1}$ by a single generalized crossing change of sign $-\epsilon_{i}$ for $i=1, \ldots, p+n$ and $U$ is the unknot.

Consider $U$ to be embedded in $\partial B^{4}=S^{3}$. Since $U$ is an unknot in $S^{3}$, it bounds an embedded disk $D \subset S^{3}$. We push $D$ into $B^{4}$ to get a disk $\Delta_{0} \subset B^{4}$ such that $\Delta_{0} \cap \partial B^{4}=U$ and $\pi_{1}\left(B^{4} \backslash \Delta_{0}\right)=\mathbb{Z}$, where the latter is generated by a meridian of $U$. 
Now, we build a 4-manifold $C$ in which $K$ bounds a disk $\Delta$ as follows. Let $C_{0}:=B^{4}$. We now build $C$ from $C_{0}$ by sequentially thickening the boundary of $C_{0}$ and attaching 2handles to the new boundary. First, we thicken the boundary $S_{0}:=\partial B^{4}$ to $S_{0} \times[0,1]$, obtaining a new 4-manifold $B_{0}$. We denote the disk $\Delta_{0} \cup(U \times I) \subset B_{0}$ by $\Delta_{1}$. The first generalized crossing change can be realized via the attachment of a $-\epsilon_{1}$-framed 2-handle $h_{1}$ along an unknot $U_{1} \subset S_{0} \times\{1\}$ with $\operatorname{lk}\left(U \times\{1\}, U_{1}\right)=0$. There is a unique orientationpreserving diffeomorphism from the new boundary $S_{1}$ resulting from this handle attachment to $S^{3}$, and after this diffeomorphism $U \times\{1\}$ is isotopic to $K_{1}$. We denote by $C_{1}$ the new 4 -manifold resulting from this handle attachment. Since attaching a \pm 1 -framed 2 -handle to the boundary of a 4-manifold along an unknot results in connect-summing a $\pm \mathbb{C P}^{2}$, we have that $C_{1} \cong C_{0} \#-\epsilon_{1} \mathbb{C P}^{2}=B^{4} \#-\epsilon_{1} \mathbb{C P}^{2}$ (here $\pm \mathbb{C P}^{2}$ denotes $\mathbb{C P}^{2}$ or $\overline{\mathbb{C P}^{2}}$, respectively). Note that $\Delta_{1}$ is still a disk in $C_{1}$ and that $\partial \Delta_{1}=K_{1}$.

Attaching a 2-handle generally adds a relation to the fundamental group of the resulting manifold, where the relation is given by the attaching map. Since the attaching circle $U_{1}$ of $h_{1}$ is trivial in $H_{1}\left(\left(S_{0} \times\{1\}\right) \backslash(U \times\{1\})\right) \cong \mathbb{Z}\left\langle\mu_{0}\right\rangle$, where $\mu_{0}$ is a meridian of $U \times\{1\} \subset S_{0} \times\{1\}$, it is also trivial in $\pi_{1}\left(B_{0} \backslash \Delta_{1}\right) \cong \mathbb{Z}\left\langle\mu_{0}\right\rangle$. Thus, we get that $\pi_{1}\left(C_{1} \backslash \Delta_{1}\right) \cong \mathbb{Z}$ as well, generated by a meridian $\mu_{1}$ of $K_{1}$.

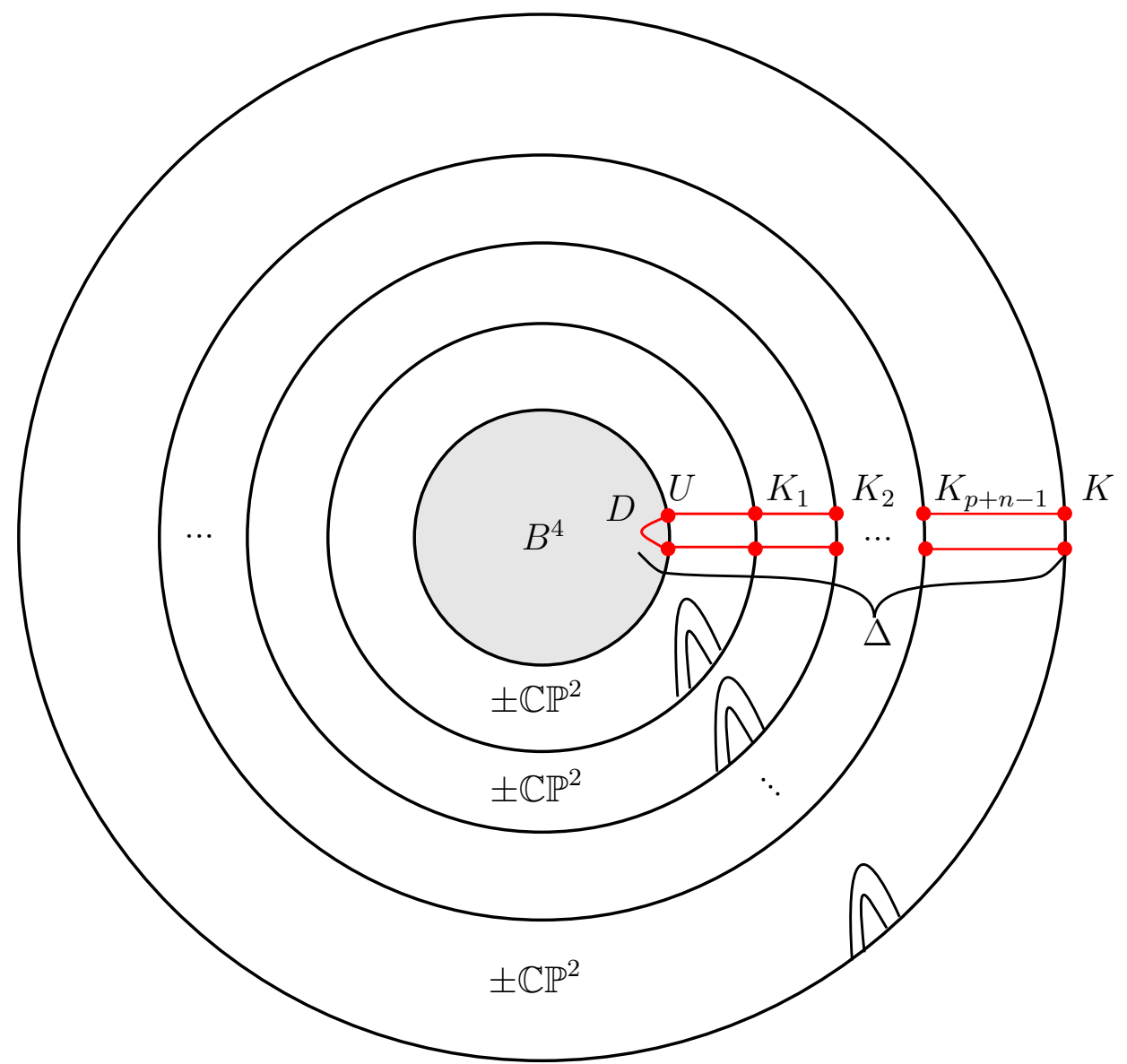

Figure 4.1. The construction of a manifold $C$ in which $K$ bounds a disk $\Delta$. 
We continue in this way to iteratively get 4 -manifolds $C_{1}, \ldots, C_{p+n}$ so that $C_{i+1}$ is obtained from $C_{i}$ by adding a collar $\partial C_{i} \times[i, i+1]$ to $\partial C_{i}$ and attaching a $-\epsilon_{i+1}$-framed 2-handle $h_{i+1}$ to $\partial C_{i} \times\{i+1\}$. At each stage, the attaching circle $U_{i+1} \subset S_{i} \times i+1$ of $h_{i+1}$ is trivial in

$$
H_{1}\left(\left(S_{i} \times\{i+1\}\right) \backslash\left(K_{i} \times\{i+1\}\right)\right) \cong \mathbb{Z}\left\langle\mu_{i}\right\rangle,
$$

where $\mu_{i}$ is a meridian of $K_{i} \times i+1$. Hence, $U_{i+1}$ is trivial in $\pi_{1}\left(B_{i} \backslash \Delta_{i+1}\right) \cong \mathbb{Z}\left\langle\mu_{i}\right\rangle$. The end result of this process is a 4 -manifold $C:=C_{p+n} \cong B^{4} \#_{n} \mathbb{C P}^{2} \#_{p} \overline{\mathbb{C P}^{2}}$ in which $K:=K_{p+n}$ bounds a disk $\Delta:=\Delta_{p+n}$ such that $\pi_{1}(C \backslash \Delta) \cong \mathbb{Z}$, generated by a meridian $\mu_{p+n}$ of $K=K_{p+n}$.

We now consider the non-degenerate intersection form $H_{2}(C, \partial C) \times H_{2}(C) \rightarrow \mathbb{Z}$ in order to show that $[\Delta]=0 \in H_{2}(C, \partial C)$. Since $H_{2}(C) \cong \mathbb{Z}^{p+n}$ is generated by the $\mathbb{C P}^{1}$ factors $\mathbb{C P}_{1}^{1}, \ldots, \mathbb{C P}_{p+n}^{1}$, where $\mathbb{C P}_{i}^{1}$ is a generator of the second homology of the $i$ th connect-summed copy of $\pm \mathbb{C P}^{2}$, we know that an element $a \in H_{2}(C, \partial C)$ is 0 if and only if $a \cdot\left[\mathbb{C P}_{i}^{1}\right]=0$ for all $i=1, \ldots, p+n$.

Let $d_{i}$ denote the disk bounded by the unknot $U_{i}$, and let $D_{i}$ denote the second $D^{2}$ factor in the $i$ th 2-handle attached to $C$. Then $\mathbb{C P}_{i}^{1}$ is homologous to

$$
\left(d_{i-1} \times\left\{i-\frac{1}{2}\right\}\right) \cup\left(U_{i} \times\left[i-\frac{1}{2}, i\right]\right) \cup\left(* \times D_{i}\right) .
$$

The only intersections of $\Delta$ with $\mathbb{C P}_{i}^{1}$ come from the intersections of $K_{i-1}$ with $d_{i}$. Since $\operatorname{lk}\left(K_{i-1}, U_{i}\right)=0$ for all $i,\left[K_{i-1}\right] \cdot\left[d_{i}\right]=0$ for all $i$. Therefore, $[\Delta]=0 \in H_{2}(C, \partial C)$. This completes the proof of the proposition.

Next, we prove a generalization of [NO15, Proposition 2.3]:

Proposition 4.2. Let $K$ be a knot in $S^{3}=\partial B^{4}$, and suppose $K$ bounds a properly embedded disk $\Delta$ in $C:=B^{4} \#_{n} \mathbb{C P}^{2} \#_{p} \overline{\mathbb{C P}^{2}}$ such that $[\Delta]=0 \in H_{2}(C, \partial C)$ and $\pi_{1}(C \backslash \Delta)=\mathbb{Z}$, generated by a meridian of $K$. Then there exists an oriented 4-manifold $W$ with boundary $\partial W=\Sigma(K)$, the branched double cover of $K$, such that

(1) $W$ is simply connected;

(2) $H_{2}(W ; \mathbb{Z}) \cong \mathbb{Z}^{2(p+n)}$;

(3) the signature of $W$ is $\sigma(W)=2(n-p)+\sigma(K)$;

(4) there exist $p+n$ pairwise disjoint classes in $H_{2}(W ; \mathbb{Z})$ represented by $p$ surfaces of self-intersection -2 and $n$ surfaces of self-intersection +2 which span a primitive sublattice; in other words, the quotient of $H_{2}(W ; \mathbb{Z})$ by this sublattice is torsion-free.

Proof. Since $\pi_{1}(C \backslash \Delta)=\mathbb{Z}$ with generator the meridian of $K$, we may take the double cover $W=\Sigma_{2}(C, \Delta)$ of $C$ branched along $\Delta$, and by definition we have $\partial W=\Sigma_{2}(K)$.

Claim. $W$ is simply connected.

Let $p:(C \widetilde{\backslash N(\Delta)}) \rightarrow C \backslash N(\Delta)$ denote the two-fold, unbranched cover of the complement of an open tubular neighborhood of $\Delta$ in $C$. Since $\pi_{1}(C \backslash \Delta) \cong \mathbb{Z}$, we have that $\pi_{1}(\widetilde{C \backslash \Delta} \cong \mathbb{Z}$ 
as well. The branched cover $W$ may be recovered from $C \widetilde{\backslash N(\Delta)}$ by gluing back a closed neighborhood $\overline{N(\Delta)} \cong D^{2} \times \Delta$ along $p^{-1}(\partial \overline{N(\Delta)}) \cong S^{1} \times \Delta$. A straightforward application of the Seifert-van Kampen theorem to $W=\widehat{C \backslash \Delta} \cup_{p^{-1}(\partial \overline{N(\Delta)})} \overline{N(\Delta)}$ shows that $\pi_{1}(W)=1$. Claim. The Euler characteristic of $W$ is $\chi(W)=2(p+n)+1$.

By a standard Mayer-Vietoris argument, we may show that

$$
H_{i}(C)= \begin{cases}\mathbb{Z}, & i=0 \\ 0, & i=1,3 \\ \mathbb{Z}^{p+n}, & i=2 \\ 0, & i=4\end{cases}
$$

where $H_{4}(C)=0$ because $C$ is a manifold with boundary. Thus, $\chi(C)=1+p+n$. We have that

$$
\chi(C)=\chi(C \backslash \Delta)+\chi(\Delta)=\chi(C \backslash \Delta)+1 .
$$

Therefore, the double cover $\widetilde{C \backslash \Delta}$ of $C \backslash \Delta$ has Euler characteristic $2(\chi(C)-1)$. Since $W=\widetilde{C \backslash \Delta} \cup_{p^{-1}(\partial \overline{N(\Delta)})} \overline{N(\Delta)}$ as above, we have that

$$
\chi(W)=2(\chi(C)-1)+1=2(p+n)+1 .
$$

Claim. The second homology of $W$ is $\mathbb{Z}^{2(p+n)}$.

Since $H_{1}(W ; \mathbb{Z})=0$, the Universal Coefficient Theorem together with the long exact cohomology sequence for $(X, \partial X)$ implies that $H^{1}(W, \partial W ; \mathbb{Z})=0$ as well. By Poincaré-Lefschetz duality, we have that $H_{3}(W ; \mathbb{Z})=0$ as well. Note that $H_{4}(W ; \mathbb{Z})=0$ since $W$ is a manifold with boundary. Now the Euler characteristic of $W$ is

$$
2 p+2 n+1=\chi(W)=1+b_{2}(W) .
$$

Therefore, $b_{2}(W)=2(p+n)$, and $H_{2}(W ; \mathbb{Z})$ is free abelian of rank $2(p+n)$. This completes the proof of the Claim.

Claim. The signature of $W$ is $\sigma(W)=2(n-p)+\sigma(K)$.

Our proof follows the proof of [CL86, Theorem 3.7]. Let $F_{-K}$ be a connected Seifert surface of the knot $-K$ with interior pushed into $-B^{4}$. Then the manifold $(\hat{C}, F):=(C, \Delta) \cup_{\left(S^{3}, K\right)}$ $\left(-B^{4}, F_{-K}\right)$ is closed. Let $\hat{W}$ denote the double cover $\Sigma_{2}(\hat{C}, F)$ of $\hat{C}$ branched over $F:=$ $\Delta \cup_{K} F_{-K}$. Then $\hat{W}=W \cup_{\Sigma_{2}(K)} X_{K}$, where $X_{K}$ is the double cover $\Sigma_{2}\left(F_{-K}\right)$ of $-B^{4}$ branched along $F_{-K}$. By [Vir73, KT76], the signature of $X_{K}$ is $-\sigma(K)$. Applying Novikov additivity, we get that

$$
\sigma(\hat{W})=\sigma(W)+\sigma\left(X_{K}\right) .
$$

Furthermore, the $G$-signature theorem [CG78, Lemma 2.1] tells us that

$$
\sigma(\hat{W})=2 \sigma(\hat{C})-\frac{1}{2}([F] \cdot[F]) .
$$

Since in this case $[\Delta]=0 \in H_{2}(C, \partial C)$, we have that $[F] \cdot[F]=0$ so

$$
\sigma(W)=2 \sigma(C)+\sigma(K) \text {. }
$$


Since $\sigma(C)=n-p$, we get that $\sigma(W)=2(n-p)+\sigma(K)$. This completes the proof of the Claim.

Claim. There exist $p+n$ pairwise disjoint classes in $H_{2}(W ; \mathbb{Z}), p$ of self-intersection -2 and $n$ of self-intersection +2 , which span a primitive sublattice.

We let $S_{i}$ be a smoothly embedded surface representing the generator of $H_{2}\left(-\epsilon_{i} \mathbb{C P}_{i}^{2}\right)$, the $i$ th summand of $C$. We define $x_{i}$ to be the homology class of the two-fold cover $\hat{S}_{i} \subset W$ of $S_{i}$ branched over $\Delta \cap S_{i}$, which is a subset of $W$. Since the $S_{i}$ are pairwise disjoint downstairs, the $\hat{S}_{i}$ are also pairwise disjoint. We show that the $x_{i}$ have self-intersection $-2 \epsilon_{i}$.

Let $S_{i}^{+}$be a push-off of $S_{i}$. Then $S_{i} \cdot S_{i}^{+}=-\epsilon_{i}$. We make the disk $\Delta$ disjoint from the (codimension-2) intersection points $S_{i} \cap S_{i}^{+}$. In the branched cover, denote the preimage of $S_{i}$ by $T_{i}$ and the preimage of $S_{i}^{+}$by $T_{i}^{+}$. Then $T_{i}^{+}$is also a push-off of $T_{i}$. The intersection points of $T_{i}$ and $T_{i}^{+}$are the preimages of the intersection points of $S_{i}$ and $S_{i}^{+}$; since the points of $S_{i} \cap S_{i}^{+}$are disjoint from the branch set, there are geometrically two intersection points of $T_{i}$ and $T_{i}^{+}$. Furthermore, the orientations upstairs give the same signs of intersection as downstairs. Therefore, $T_{i} \cdot T_{i}^{+}=-2 \epsilon_{i}$.

The proof of [NO15, Proposition 2.3] applies to our case to show that these classes span a primitive sublattice. This completes the proof of the Proposition.

Remark 4.3. The proof of Proposition 4.2 is very similar to the proof of [NO15, Proposition 2.3], with the caveat that Nagel and Owens use only -1-generalized crossing changes in order to unknot $K$, no matter the signs of the crossings of $K$ that need to be changed (see Figure 4.2). The diagram on the right side of Figure 4.2 is not a generalized crossing change diagram, since $\operatorname{lk}(K, U) \neq 0$. Therefore, we must assume that $K$ can be unknotted only by positive generalized crossing changes.

From Propositions 4.1 and 4.2, we derive a theorem analogous to [NO15, Theorem 1], but requiring the additional condition that the signature of the knot $K$ is 0 :

Theorem 4.4. Let $K \subset S^{3}$ be an oriented knot with signature 0 which can be unknotted by $p$ generalized crossing changes, all of sign +1 . Then the double cover $Y:=\Sigma(K)$ of $S^{3}$ branched along $K$ bounds a smooth, simply connected, negative definite 4-manifold $W$ with $\mathrm{H}_{2}(W ; \mathbb{Z}) \cong \mathbb{Z}^{2 p}$. Moreover, $\mathrm{H}_{2}(W ; \mathbb{Z})$ contains $p$ pairwise disjoint homology classes of self-intersection -2 which span a primitive sublattice.

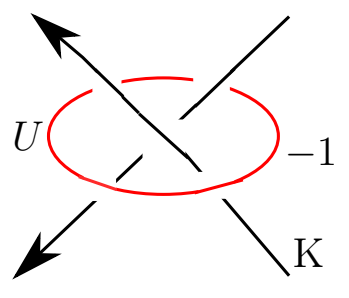

$$
\operatorname{lk}(K, U)=0
$$

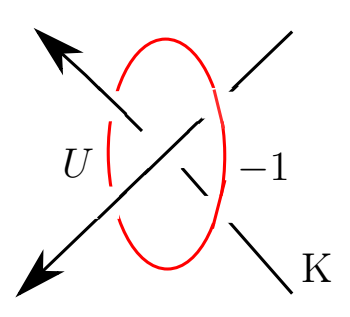

$\operatorname{lk}(K, U)= \pm 2$

Figure 4.2. No matter the sign of the crossing to be changed, Nagel and Owens [NO15] may perform only -1-generalized crossing changes in order to do so. 
Proof. By Proposition 4.1, $K$ bounds a disk $\Delta$ in a manifold $C \cong B^{4} \#_{p} \mathbb{C P}^{2}$ such that $[\Delta]=0 \in H_{2}(C, \partial C)$ and $\pi_{1}(C \backslash \Delta)=\mathbb{Z}\langle\mu\rangle$, where $\mu$ is a meridian of $K$. By Proposition 4.2, the double cover $W:=\Sigma_{2}(C, \Delta)$ of $C$ branched over $\Delta$ is simply connected, has $H_{2}(W ; \mathbb{Z}) \cong$ $\mathbb{Z}^{2 p}$, and contains $p$ pairwise disjoint homology classes of self-intersection -2 which span a primitive sublattice. Moreover, the signature of $W$ is $\sigma(W)=-2 p+\sigma(K)=-2 p$, so that $W$ is negative definite.

Note 4.5. If instead $K$ can be unknotted using $n$ generalized crossing changes, all of sign -1 , Theorem 4.4 applied to $-K$ shows that the double cover $-Y$ of $S^{3}$ branched along $-K$ bounds a smooth negative definite 4 -manifold $W$ with $b_{1}(W)=0, b_{2}(W)=2 n$, and such that $H_{2}(W ; \mathbb{Z})$ contains $n$ pairwise disjoint surface classes of self-intersection -2 which span a primitive sublattice.

In the rest of this paper, we will say $t u(K)= \pm 1$ if $K$ can be unknotted by a single \pmgeneralized crossing change. If $\sigma(K)=0$ and $t u(K)= \pm 1$, we can always get a negative definite 4-manifold $W$ bounding $\pm \Sigma(K)$ : if $K$ can be unknotted by a positive generalized crossing change, then we get a negative definite $W$ bounding $+\Sigma(K)$, and if $K$ can be unknotted by a negative generalized crossing change, then we get a negative definite $W$ bounding $-\Sigma(K)$. Moreover, the intersection form on $W$ is represented by a definite $2 \times 2$ matrix $Q$. For an $n \times n$ matrix $M$, we denote by $\Gamma_{M}$ the group $\mathbb{Z}^{n} / M\left(\mathbb{Z}^{n}\right)$. With this terminology established, we may state the following corollary of Theorem 4.1, which simplifies our computations:

Corollary 4.6. Let $K$ be an alternating knot such that $t u(K)= \pm 1$ and $\sigma(K)=0$. We use the convention that $\operatorname{det} K=\left|\Delta_{K}(-1)\right|>0$. Let $G$ be the negative definite Goeritz matrix obtained from an alternating diagram for $\pm K$. Then there exists a negative definite matrix of the form

$$
Q=\left(\begin{array}{cc}
-\frac{\operatorname{det} K+1}{2} & 1 \\
1 & -2
\end{array}\right)
$$

so that $\pm Y= \pm \Sigma(K)$ bounds a negative definite 4-manifold with intersection form $Q$. Moreover, there is an isomorphism $\phi: \Gamma_{Q} \rightarrow \Gamma_{G}$ such that

$$
\begin{aligned}
& m_{Q}(g) \leq m_{G}(\phi(g)) \\
& m_{Q}(g) \equiv m_{G}(\phi(g)) \quad \bmod 2
\end{aligned}
$$

for all $g \in \Gamma_{Q}$.

Proof. By Theorem 4.4, $\pm Y$ bounds a negative definite 4-manifold with intersection form represented by

$$
P=\left(\begin{array}{cc}
a & b \\
b & -2
\end{array}\right)
$$

for some $a, b \in \mathbb{Z}$. By Theorem 2.2, there must exist isomorphisms

$$
\Gamma_{P} \stackrel{\phi}{\cong} \operatorname{Spin}^{c}( \pm Y) \cong H^{2}(Y ; \mathbb{Z}) \stackrel{\text { P.D. }}{\cong} H_{1}(Y ; \mathbb{Z})
$$

where the isomorphism labeled "P.D." is from Poincaré duality and the order of $H_{1}(Y ; \mathbb{Z})$ is equal to $\operatorname{det} K$. The matrix $P$ presents the group $\mathbb{Z} /(\operatorname{det} P) \mathbb{Z}$. Therefore, we must have $\operatorname{det} P= \pm \operatorname{det} K$. Since $\operatorname{det} K$ is odd, we have that

$$
b^{2} \equiv-2 a-b^{2}=\operatorname{det} P \equiv \operatorname{det} K \equiv 1 \bmod 2
$$


and hence $b$ is odd. Therefore, we can use simultaneous row and column operations to change $P$ into a matrix of form

$$
Q=\left(\begin{array}{cc}
a & 1 \\
1 & -2
\end{array}\right)
$$

Since $Q$ is negative definite, $\operatorname{det} Q \geq 0$, so that we must have $\operatorname{det} Q=+\operatorname{det} K$. Therefore, $a=-(\operatorname{det} K+1) / 2$. It follows from Theorem 3.1 that $m_{Q}(g) \leq m_{G}(g)$ and that the two are congruent modulo 2. The corollary follows.

Note 4.7. Ozsváth and Szabó used a similar process to obstruct knots from having unknotting number 1 in [OS05], although their isomorphisms $\phi$ were also required to satisfy a "symmetry" condition which is not necessarily satisfied in our case. In [OS05, Corollary 1.3], Ozsváth and Szabó computed the $m_{Q}$ and $m_{G}$ for various knots to determine whether there exist isomorphisms $\phi$ of the type given in Corollary 4.6. The only knot with signature 0 which had its unknotting number determined by Ozsváth and Szabó for which the untwisting number was unknown and for which the "symmetry" condition was not necessary is $10_{68}$. In this way, we get from their computations that $t u\left(10_{68}\right)=2=u\left(10_{68}\right)$, even though $u_{a}\left(10_{68}\right)=1$.

\section{EXAMPles}

In this section, we will prove Theorems 1.3 and 1.4 using Corollary 4.6. Following Ozsváth and Szabó in [OS05], we will refer to an isomorphism $\phi$ satisfying (4.3) as a positive matching and an isomorphism $\phi$ satisfying (4.4) as an even matching. We obstruct the existence of positive, even matchings for each of the cases listed in Theorem 1.3. We illustrate the proof that $t u\left(10_{68}\right)=2$; the remaining knots are obstructed from having untwisting number +1 and/or -1 similarly.

Example 5.1. Although Ozsváth and Szabó have already verified in [OS05] that $\Sigma\left(10_{68}\right)$ cannot bound a 4-manifold with intersection form

$$
Q=\left(\begin{array}{cc}
-29 & 1 \\
1 & -2
\end{array}\right)
$$

as it would have to if $t u\left(10_{68}\right)=1$, we replicate the computation below. The knot $10_{68}$ has $\sigma\left(10_{68}\right)=0, \operatorname{det} 10_{68}=57$, and Goeritz matrix

$$
G=\left(\begin{array}{ccccccc}
-4 & 1 & 1 & 0 & 0 & 1 & 0 \\
1 & -3 & 0 & 0 & 1 & 0 & 0 \\
1 & 0 & -2 & 1 & 0 & 0 & 0 \\
0 & 0 & 1 & -2 & 1 & 0 & 0 \\
0 & 1 & 0 & 1 & -3 & 0 & 1 \\
1 & 0 & 0 & 0 & 0 & -2 & 1 \\
0 & 0 & 0 & 0 & 1 & 1 & -2
\end{array}\right)
$$

The values of $m_{G} \bmod 2$ are

$\begin{array}{rrrrrrrrr}0 & 98 / 57 & 50 / 57 & 28 / 19 & 86 / 57 & 56 / 57 & 36 / 19 & 14 / 57 & 2 / 57 \\ 24 / 19 & 110 / 57 & 2 / 57 & 30 / 19 & 32 / 57 & 56 / 57 & 16 / 19 & 8 / 57 & 50 / 57 \\ 20 / 19 & 2 / 3 & 98 / 57 & 4 / 19 & 8 / 57 & 86 / 57 & 6 / 19 & 32 / 57 & 14 / 57 \\ 26 / 19 & 110 / 57 & 110 / 57 & 26 / 19 & 14 / 57 & 32 / 57 & 6 / 19 & 86 / 57 & 8 / 57 \\ 4 / 19 & 98 / 57 & 2 / 3 & 20 / 19 & 50 / 57 & 8 / 57 & 16 / 19 & 56 / 57 & 32 / 57\end{array}$


$30 / 19 \quad 2 / 57 \quad 110 / 57 \quad 24 / 19 \quad 2 / 57 \quad 14 / 57 \quad 36 / 19 \quad 56 / 57 \quad 86 / 57$

$28 / 19 \quad 50 / 57 \quad 98 / 57$.

If $\Sigma\left(10_{68}\right)$ bounded a 4-manifold $W$ as in Corollary 4.6 , the matrix

$$
Q=\left(\begin{array}{cc}
a & 1 \\
1 & -2
\end{array}\right)
$$

representing the intersection form on $W$ would have determinant $-2 a-1=\operatorname{det}\left(10_{68}\right)=57$, so that $a=-29$ and

$$
Q=\left(\begin{array}{cc}
-29 & 1 \\
1 & -2
\end{array}\right)
$$

In this case, the values of $m_{Q} \bmod 2$ are

$\begin{array}{rrrrrrrrr}0 & 112 / 57 & 106 / 57 & 32 / 19 & 82 / 57 & 64 / 57 & 14 / 19 & 16 / 57 & 100 / 57 \\ 22 / 19 & 28 / 57 & 100 / 57 & 18 / 19 & 4 / 57 & 64 / 57 & 2 / 19 & 58 / 57 & 106 / 57 \\ 12 / 19 & 4 / 3 & 112 / 57 & 10 / 19 & 58 / 57 & 82 / 57 & 34 / 19 & 4 / 57 & 16 / 57 \\ 8 / 19 & 28 / 57 & 28 / 57 & 8 / 19 & 16 / 57 & 4 / 57 & 34 / 19 & 82 / 57 & 58 / 57 \\ 10 / 19 & 112 / 57 & 4 / 3 & 12 / 19 & 106 / 57 & 58 / 57 & 2 / 19 & 64 / 57 & 4 / 57 \\ 18 / 19 & 100 / 57 & 28 / 57 & 22 / 19 & 100 / 57 & 16 / 57 & 14 / 19 & 64 / 57 & 82 / 57 \\ 32 / 19 & 106 / 57 & 112 / 57 . & & & & & & \end{array}$

These lists are not identical (in particular, there is a $112 / 57$ in the $m_{Q}$ list but not in the $m_{G}$ list), so there are no even matchings here and $t u\left(10_{68}\right) \neq+1$.

The Goeritz matrix for $-10_{68}$ is

$$
G^{\prime}=\left(\begin{array}{ccc}
-3 & 1 & 0 \\
1 & -5 & 3 \\
0 & 3 & -6
\end{array}\right)
$$

the values of $m_{G^{\prime}}$ are

$\begin{array}{rrrrrrrrr}0 & 4 / 57 & 16 / 57 & 12 / 19 & -50 / 57 & -14 / 57 & 10 / 19 & -32 / 57 & 28 / 57 \\ -6 / 19 & -56 / 57 & 28 / 57 & 2 / 19 & -8 / 57 & -14 / 57 & -4 / 19 & -2 / 57 & 16 / 57 \\ -24 / 19 & -2 / 3 & 4 / 57 & -20 / 19 & -2 / 57 & -50 / 57 & -30 / 19 & -8 / 57 & -32 / 57 \\ -16 / 19 & -56 / 57 & -56 / 57 & -16 / 19 & -32 / 57 & -8 / 57 & -30 / 19 & -50 / 57 & -2 / 57 \\ -20 / 19 & 4 / 57 & -2 / 3 & -24 / 19 & 16 / 57 & -2 / 57 & -4 / 19 & -14 / 57 & -8 / 57 \\ 2 / 19 & 28 / 57 & -56 / 57 & -6 / 19 & 28 / 57 & -32 / 57 & 10 / 19 & -14 / 57 & -50 / 57 \\ 12 / 19 & 16 / 57 & 4 / 57 . & & & & & & \end{array}$

Using a Python program, we check all possible isomorphisms $\phi$ and find that there are no positive, even matchings between the values of $m_{Q}$ and the values of $m_{G^{\prime}}$. Therefore, $t u\left(10_{68}\right) \neq-1$. Since $u\left(10_{68}\right)=2$, we must have that $t u\left(10_{68}\right)=2$ as well.

\section{OzSVÁth-SZABÓ $\tau$ INVARIANT AND RASMUSSEn $s$ INVARIANT OBSTRUCTIONS TO $p$-UNTWISTING NUMBER}

In this section, we investigate $p$-generalized crossing changes for fixed $p$ in order to prove Theorem 1.5. 
Every $p$-generalized crossing change consists of $p(p-1)+p^{2}=p(2 p-1)$ standard crossing changes. Thus, for every positive integer $p$ and every knot $K \subset S^{3}$, if $t u_{p}(K) \leq n$, then there is an unknotting sequence consisting of $p n(2 p-1)$ crossing changes, so that

$$
u(K) \leq p(2 p-1) t u_{p}(K)
$$

whence

$$
|\tau(K)| \leq u(K) \leq p(2 p-1) t u_{p}(K)
$$

Thus, it is possible to use the $\tau$ invariant to get lower bounds on $t u_{p}$ for all $p$. These bounds may be useful in distinguishing $t u_{p}$ from $t u_{q}$ for $p \neq q$. However, we may obtain a stronger bound using the smooth 4-genus as follows. While visiting Mark Powell at the Max Planck Institute, he suggested this theorem and told me an outline of a somewhat more complicated proof. It is similar to the proof of Powell and coauthors T. Cochran, S. Harvey, and A. Ray that the $\tau$ and $s$ invariants give lower bounds for their bipolar metrics (to appear in a future paper). The following, simpler proof involving the 4-genus was suggested by the referee.

Theorem 6.1. If $K$ can be unknotted by $k$ generalized crossing changes, where the ith change is performed on $2 q_{i}$ strands, then

$$
g_{4}(K) \leq \sum_{i=1}^{k} q_{i}^{2}
$$

Proof. Suppose that $K$ may be unknotted via $k$ generalized crossing changes. Then there is a sequence of $k$ generalized crossing changes taking $K$ to $U$ :

$$
K=K_{0} \stackrel{q_{1} \text {-gcc }}{\longrightarrow} K_{1} \stackrel{q_{2} \text {-gcc }}{\longrightarrow} \ldots \stackrel{q_{k-1} \text {-gcc }}{\longrightarrow} K_{k-1} \stackrel{q_{k} \text {-gcc }}{\longrightarrow} K_{k}=U .
$$

for which $K_{i}$ is obtained from $K_{i-1}$ by a single $q_{i}$-generalized crossing change for $i=1, \ldots, k$. Let $D_{i}$ be the disk bounded by the unknot $U_{i}$ on which the $i$ th $q_{i}$-generalized crossing change is performed.

First, note that we can isotope $D_{i}$ so that the strands of $K_{i-1}$ pass through it as in Figure 6.1. The strands passing through $D_{i}$ are oriented in two different ways; we separate the $q_{i}$ strands of each orientation as in the figure. Let us arbitrarily call one group of $q_{i}$ strands (say, the ones on the top of the figure) "left-oriented" and the other group "right-oriented". Hence, we may assume without loss of generality that we have a local picture as in Figure 6.1 .

A $q_{i}$-generalized crossing change can be undone by changing $q_{i}\left(2 q_{i}-1\right)$ crossings; one changes precisely one crossing between the $i$ th and $j$ th strands $\left(s_{i}\right.$ and $s_{j}$ ) for each $1 \leq i<j \leq 2 q_{i}$. Since $q_{i}$ of the strands are oriented in one direction and $q_{i}$ in the other, $q_{i}^{2}$ of these crossing changes occur between strands oriented in opposite directions and $q_{i}\left(q_{i}-1\right)$ occur between strands oriented in the same direction (see Figure 6.2 for an illustration in the case of a 4-generalized crossing change). Thus, $q_{i}^{2}$ of the crossing changes have one sign, and $q_{i}^{2}-q_{i}$ have the other sign. Therefore, $K$ can be unknotted by changing $P$ positive crossings and 
$N$ negative crossings, where

$$
\max \{P, N\} \leq \sum_{i=1}^{k} q_{i}^{2} .
$$

However, it is well known, for instance by the argument in the third paragraph of the introduction of [Owe10], that if $K$ can be unknotted by changing $P$ positive crossings and $N$ negative crossings, then $g_{4}(K) \leq \max \{P, N\}$.

Since the Ozsváth-Szabó $\tau$ invariant and Rasmussen $s$ invariant give lower bounds on the slice genus of any knot, we immediately get the following:

Corollary 6.2. Let $K$ be a knot which can be converted to the unknot via $k$ generalized crossing changes, where the ith generalized crossing change is performed on $2 q_{i}$ strands for $i=1, \ldots, k$. Then

$$
|\tau(K)| \leq \sum_{i=1}^{k} q_{i}^{2}
$$

and

$$
\frac{|s(K)|}{2} \leq \sum_{i=1}^{k} q_{i}^{2} .
$$

This corollary gives rise to a method for distinguishing $t u_{q}(K)$ from $t u_{p}(K)$ for some $p, q>1$. Suppose that $t u_{q}(K) \leq n$. Then there exists an untwisting sequence for $K$ consisting of $n$

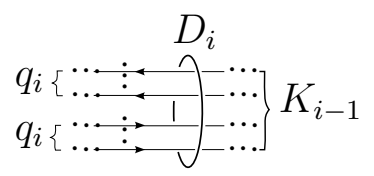

FiguRE 6.1. The result of the isotopy on $D_{i}$ and the strands of $K_{i-1}$. We call the strands on the top left-oriented and those on the bottom right-oriented.

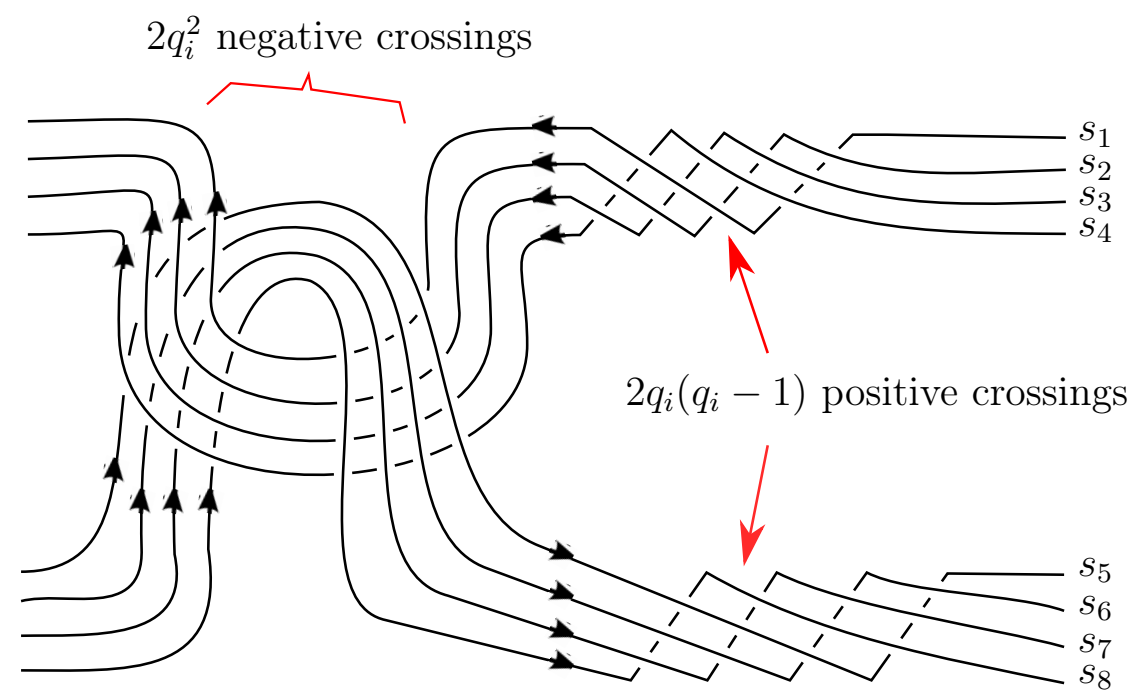

Figure 6.2. Two sets of four strands twisted around each other at a positive 4-generalized crossing change. 
generalized crossing changes on $2 p_{i}$ strands each, where $i=1, \ldots, n$ and $p_{i} \leq q$ for all $i$. Applying the corollary, we get that

$$
|\tau(K)| \leq \sum_{i=1}^{n} p_{i}^{2} \leq \sum_{i=1}^{n} q^{2}=n q^{2}
$$

so that we must have

$$
n \geq \frac{|\tau(K)|}{q^{2}}
$$

and similarly for $|s(K)| / 2$ in place of $|\tau(K)|$. We thus obtain the following obstruction to $t u_{q}(K)=n$ :

Corollary 6.3. For all integers $q \geq 1$ and all knots $K \subset S^{3}$,

$$
t u_{q}(K) \geq \frac{|\tau(K)|}{q^{2}} \text { and } t u_{q}(K) \geq \frac{|s(K)|}{2 q^{2}} .
$$

Note 6.4. The above obstruction shows that $|\tau(K)| \leq p^{2} \cdot t u_{p}(K)$ for all $K$, which is stronger than the obstruction $|\tau(K)| \leq p(2 p-1) t u_{p}(K)$ given by representing a $p$-generalized crossing change as $p(2 p-1)$ standard crossing changes.

Example 6.5. Let $K_{p^{3}}$ denote the $\left(p^{3}, 1\right)$-cable of a knot $K$ with $u(K)=1=\tau(K)=g(K)$ (one example is the right-handed trefoil knot). We know from [Inc16, Section 5.1] that $t u_{p^{3}}\left(K_{p^{3}}\right)=1$ and that $\tau\left(K_{p^{3}}\right)=p^{3}$. However, the above result shows that

$$
t u_{p}\left(K_{p^{3}}\right) \geq \frac{\left|\tau\left(K_{p^{3}}\right)\right|}{p^{2}}=p .
$$

for all integers $p \geq 1$. Hence

$$
t u_{p}\left(K_{p^{3}}\right)-t u_{p^{3}}\left(K_{p^{3}}\right)=t u_{p}\left(K_{p^{3}}\right)-1 \geq p-1 \stackrel{p \rightarrow \infty}{\longrightarrow} \infty .
$$

\section{REFERENCES}

[Ada94] Colin C. Adams, The Knot Book, American Mathematical Soc., 1994.

[CG78] Andrew J. Casson and Cameron McA Gordon, On slice knots in dimension three, Proceedings of Symposia in Pure Mathematics 32 (1978).

[CL86] Tim D. Cochran and W. B. Raymond Lickorish, Unknotting information from 4-manifolds, Transactions of the American Mathematical Society 297 (1986), no. 1, 125-142.

[Inc16] Kenan Ince, The untwisting number of a knot, Pacific Journal of Mathematics (accepted) (2016), arXiv: math/1507.04386.

[KM93] Peter B. Kronheimer and Tomasz S. Mrowka, Gauge theory for embedded surfaces, I, Topology 32 (1993), no. 4, 773-826.

[KM95] _ Gauge theory for embedded surfaces, II, Topology 34 (1995), no. 1, 37-97.

[KT76] Louis H. Kauffman and Laurence R. Taylor, Signature of links, Transactions of the American Mathematical Society 216 (1976), 351-365.

[Liv02] Charles Livingston, The slicing number of a knot, Algebr. Geom. Topol 2 (2002), 1051-1060.

[McC13] Duncan McCoy, Alternating knots with unknotting number one, arXiv: math/1312.1278.

[MD88] Yves Mathieu and Michel Domergue, Chirurgies de Dehn de pente \pm 1 sur certains næuds dans les 3-variétés, Mathematische Annalen 280 (1988), no. 3, 501-508.

[Mon76] José M. Montesinos, Three manifolds as 3-fold branched covers of $S^{3}$, Quart. J. Math. Oxford 2 (1976), no. 27, 85-94.

[Mur90] Hitoshi Murakami, Algebraic unknotting operation, Proceedings of the Second Soviet-Japan Symposium of Topology 8 (1990), 283-292. 
[NO15] Matthias Nagel and Brendan Owens, Unlinking information from 4-manifolds, Bull. London Math. Soc. (accepted) 47 (2015), no. 6, 964-979.

[OS03a] Peter Ozsváth and Zoltán Szabó, Absolutely graded Floer homologies and intersection forms for four-manifolds with boundary, Advances in Mathematics 173 (2003), no. 2, 179-261.

[OS03b] _ On the Floer homology of plumbed three-manifolds, Geometry \& Topology 7 (2003), no. 1, $185-224$.

[OS05] _ Knots with unknotting number one and Heegaard Floer homology, Topology 44 (2005), no. $4,705-745$.

[Owe08] Brendan Owens, Unknotting information from Heegaard Floer homology, Advances in Mathematics 217 (2008), no. 5, 2353-2376.

[Owe10] - On slicing invariants of knots, Transactions of the American Mathematical Society 362 (2010), no. 6, 3095-3106.

[OweS13] Brendan Owens and Sašo Strle, Immersed disks, slicing numbers and concordance unknotting numbers, Comm. Anal. Geom. (accepted) (2013).

[Rol76] Dale Rolfsen, Knots and Links, AMS Chelsea Pub., 1976.

[Vir73] Oleg J. Viro, Branched coverings of manifolds with boundary and link invariants, I, Mathematics of the USSR-Izvestiya 7 (1973), no. 6, 1239. 